\title{
Skin Effect Modeling Based on a Differential Surface Admittance Operator
}

\author{
Daniël De Zutter, Fellow, IEEE, and Luc Knockaert, Senior Member, IEEE
}

\begin{abstract}
An important issue in high-frequency signal integrity prediction is the modeling of the skin effect of thick conductors. A new differential surface admittance concept is put forward allowing to replace the conductor by equivalent electric surface currents and to replace the material of the conductor by the material of the background medium the conductor is embedded in. This new concept is studied in detail for the two-dimensional TM case starting from the Dirichlet eigenfunctions of the cross section. Detailed expressions are derived for the important practical case of a rectangular cross section. Next, the differential surface admittance operator is exploited to determine the resistance and inductance matrices of a set of multiconductor lines. A first set of numerical results provides the reader with some insight into the behavior of the surface admittance matrix. A second set of results demonstrates the correctness and versatility of the new approach to determine inductance and resistance matrices.
\end{abstract}

Index Terms - Inductance matrix, resistance matrix, skin effect, surface admittance.

\section{INTRODUCTION}

$\mathbf{F}$ OR CLOCK rates in the gigahertz range and for associated rise times of the order of $100 \mathrm{ps}$, signal integrity predictions on RF boards, packages, and on-chip interconnects are increasingly dependent on dedicated electronic design automation (EDA) tools offering the combination of powerful circuit simulators and accurate electromagnetic simulators. For an overview of recent efforts in the combined use of circuit analysis and full-wave electromagnetic analysis, we refer the reader to a recent special issue of this TRANSACTIONS [1]. Moreover, the evolution toward smaller chip features and increasing clock rates continues as the International Technology Roadmap for Semiconductors (ITRS) ${ }^{1}$ predicts that the smallest on-chip features will shrink from $150 \mathrm{~nm}$ in 2003 to $50 \mathrm{~nm}$ by 2012, while the clock rate will increase from 1.5 to $10 \mathrm{GHz}$.

One very important issue in the representation of the signal conductors and their coupling is the correct modeling of the so-called skin effect. When considering the current distribution in the cross section of a conductor of width $w$, thickness $t(w \geq t)$, and conductivity $\sigma$, one can roughly distinguish between three frequency ranges. In the low-frequency range, the

\footnotetext{
Manuscript received July 5, 2004; revised January 6, 2005. This work was supported in part by a grant from the Institute for the Promotion of Innovation by Science and Technology in Flanders for a joint project with Agilent Technologies.

The authors are with the Department of Information Technology, Ghent University, B-9000 Gent, Belgium (e-mail: daniel.dezutter@ugent.be).

Digital Object Identifier 10.1109/TMTT.2005.852766
}

${ }^{1}$ The ITRS Public Home Page. [Online]. Available: http://public.itrs.net skin depth $\delta$ is much larger than both $w$ and $t$. The current distribution in the conductor is then governed by the solution of the Laplace equation with zero normal derivative at the outer conductor surface. For a conductor with invariant cross section, e.g., in the $z$-direction, this, of course, leads to a uniform current distribution with associated per unit length dc resistance $(1 / \sigma w t) \Omega \mathrm{m}^{-1}$. Recall that the skin depth $\delta$ for nonmagnetic conductors is given by $\delta=\sqrt{\left(2 / \omega \mu_{0} \sigma\right)}$ with $\omega=2 \pi f$ being the angular frequency. With increasing frequency, inductive effects come into play, pushing the currents toward the surface of the conductor and leading to an increase of the resistance and a decrease of the internal inductance. This is the case when the skin depth becomes comparable to the (smallest) dimension of the conductor's cross section (at intermediate frequencies). Moreover, when several closely spaced conductors are considered (possibly in the presence of a ground plane), this current redistribution in each conductor is also influenced by the nearby presence of the other conductors. Only for the highest frequencies and provided the skin depth becomes much smaller than both $w$ and $t$, the well-known skin effect occurs. In this case, the current is flowing in a small surface layer and the behavior of the conductor is usually described in terms of the surface impedance $Z_{s}=\sqrt{\left(j \omega \mu_{0} / \sigma\right)}=((1+j) / \sigma \delta)[2]$.

It is clear that accurate electromagnetic modeling tools need to correctly account for the redistribution of the conductor current. This has, of course, been recognized by many authors and numerous publications address this so-called current crowding problem, see e.g., [3]-[6]. We would especially like to draw the attention of the reader to [6]. In that paper, the current crowding problem is treated in the context of the partial-element equivalent-circuit (PEEC) method and the introduction of that paper provides a good overview of previous research and of the physics of the problem. Generally speaking, one has to completely solve Maxwell's equations inside the conductor, while simultaneously taking into account the outside field problem. To circumvent this coupled problem, planar electromagnetic solvers use the surface impedance [7] concept to relate the current at each point of the conductor's surface to the tangential electric field at the same point. A typical expression for this surface impedance is $Z_{\text {surf }}=Z_{s} \operatorname{coth} \sqrt{j \omega \mu_{o} \sigma} t$ with $Z_{s}$ as defined above. This surface impedance yields a dc value of $(1 / \sigma t)$ and a high-frequency value of $Z_{s}$. Acceptable results are obtained at low and high frequencies if and only if $t \ll w$. This simple model is no longer correct when the aspect ratio $w / t$ of the conductor is of the order of unity. Especially for on-chip interconnections, this is precisely the range of aspect ratios we are most interested in. Moreover, the coth behavior describing 
the current crowding in going from the low-frequency regime to the high-frequency regime is rather heuristic.

Similar to the approach in [6], the purpose of this paper is to provide a surface admittance description of the conductor. At each frequency, this description associates a fictitious electric surface current density $\mathbf{J}_{s}(\mathbf{r}, \omega)$ at each point $\mathbf{r}$ on the surface of the conductor to the tangential electric fields $\mathbf{E}_{\tan }\left(\mathbf{r}^{\prime}, \omega\right)$ at every other point on the surface, i.e., we cast the problem in a surface admittance operator format. When combined, for example, with the method of moments (MoM), this operator yields a surface admittance matrix. The operator allows to replace each conductor by equivalent surface currents and to replace the conductor medium by the medium of the material layer it is embedded in. The remaining field problem can then be solved by solely considering the interactions between the equivalent surface currents. It should be noted that the admittance operator description only depends on the geometry of each individual conductor and on the frequency and does not depend on the presence of other conductors.

In Section II, the general idea behind the differential admittance concept is outlined. We restrict ourselves to two-dimensional (2-D) configurations and to the TM case, i.e., the configuration is invariant in the $z$-direction and currents are flowing in this direction. In [6], the TM case is treated using a finite-difference solution of the Helmholtz equation in the conductor's cross section. In this paper, we opt for an approach based on the Dirichlet-Neumann operator. It turns out that this approach yields analytical results in the important practical case of a conductor with rectangular cross section (and, indeed, also for a circular cross section). The theoretical derivations leading to the differential surface admittance operator based on the Dirichlet eigenfunctions of the cross section are given in Section III. In Section IV, the general theory is applied to conductors with rectangular cross section and an explicit MoM-Galerkin admittance matrix for this problem is derived. In Section V, this admittance matrix description is used to determine the resistance and inductance matrices of a set of parallel conductors by means of an electric field integral equation (EFIE). Section VI presents two sets of numerical results. The results of Section VI-A are intended to provide the reader with some insight into the behavior of the differential surface impedance matrix. The examples of Section VI-B demonstrate the correctness and versatility of the proposed concepts for the determination of the inductance and resistance matrices of two-dimensional interconnect structures. Particular attention is devoted to comparison with previously published approaches and data. Finally, Section VII presents a number of conclusions and avenues for future work.

\section{Differential Surface Admittance ConcePt}

Consider, in the case of time-harmonic ( $e^{j \omega t}$ dependence) transverse magnetic polarization, the electric field $E_{z}$ inside a simply connected imperfectly conducting nonmagnetic cylinder with homogeneous cross section $S$, as in Fig. 1. The conductor is characterized by its constitutive parameters $\epsilon, \mu_{0}$, and $\sigma$. Further suppose that the conductor is embedded in a planar stratified medium (the extension to a more general piecewise homo-

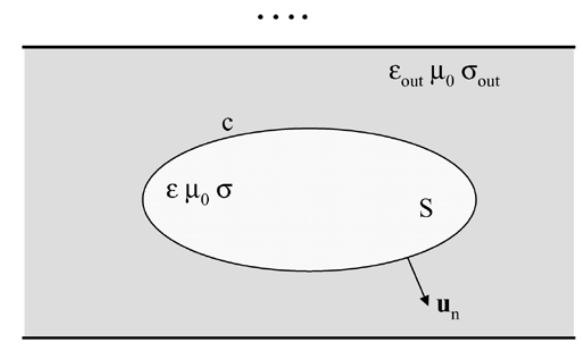

\section{$\cdots \cdots$}

Fig. 1. Cross section $S$ with boundary $c$ of a conducting cylinder embedded in a layered medium.

geneous medium is straightforward). The particular layer the conductor is embedded in is characterized by the constitutive parameters $\epsilon_{\text {out }}, \mu_{0}$, and $\sigma_{\text {out }}$. The reasonings put forward in the sequel remain valid for the limiting case whereby the conductor is exactly located on top of a layer. However, when the conductor is partly contained in one layer and partly in another, the conductor will have to be divided in two separate parts, as correctly remarked by one of the reviewers. Inside $S, E_{z}$ satisfies

$$
\nabla^{2} E_{z}+k^{2} E_{z}=0
$$

with

$$
k=\sqrt{\omega \mu_{0}(\omega \epsilon-j \sigma)} .
$$

On the boundary $c$ of $S$, we have that

$$
H_{t}=\frac{1}{j \omega \mu_{0}} \partial_{n} E_{z}
$$

with the index $t$ referring to the tangential component of the magnetic field. The expression $\partial_{n} E_{z}$ stands for the limit of the normal derivative of the electric field tending from the inside of the cylinder to $c$. We can rewrite (3) as [8]

$$
H_{t}=[\mathrm{Y}] \cdot E_{z}=\frac{1}{j \omega \mu_{0}} \mathcal{D}_{k} \cdot E_{z} .
$$

[Y] is the surface admittance operator and $\mathcal{D}_{k}$ is the Dirichlet-Neumann operator, mapping the values of the field on $c$ to the values of the normal derivatives of the field on $c$. When $k^{2}$ is not a Dirichlet eigenvalue for $S$, it is known that the Dirichlet-Neumann operator $\mathcal{D}_{k}$ is a self-adjoint pseudodifferential operator of order 1 [9].

Now suppose that the constitutive parameters of the conducting cylinder are replaced by those of the medium outside the conductor, in particular, of the material layer the conductor is embedded in. The corresponding fictitious electric field in $S$, $E_{z 0}$ now satisfies

$$
\nabla^{2} E_{z 0}+k_{\text {out }}^{2} E_{z 0}=0
$$

with

$$
k_{\mathrm{out}}=\sqrt{\omega \mu_{0}\left(\omega \epsilon_{\mathrm{out}}-j \sigma_{\mathrm{out}}\right)} .
$$




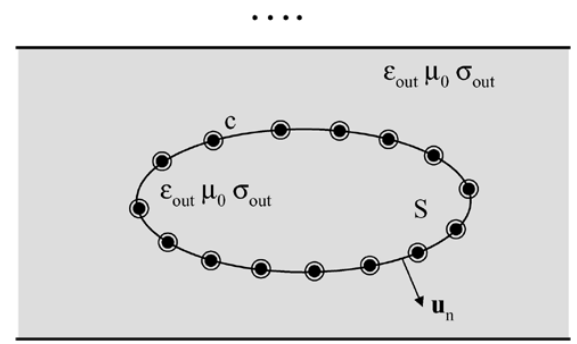

...

Fig. 2. Equivalent longitudinal currents replacing the conductor of Fig. 1 with the material $\epsilon, \mu_{0}, \sigma$ of the conductor replaced by the material $\epsilon_{\text {out }}, \mu_{0}, \sigma_{\text {out }}$ of the layer it is embedded in.

On the boundary $c$ of $S$, we now have that

$$
H_{t 0}=\frac{1}{j \omega \mu_{0}} \partial_{n} E_{z 0}
$$

and in the same vein as above,

$$
H_{t 0}=\frac{1}{j \omega \mu_{0}} \mathcal{D}_{k_{\text {out }}} \cdot E_{z 0} .
$$

If we want to replace the conductor by the material of its surrounding layer, in this way restoring the planar stratified nature of the medium and undoing the discontinuity in conductivity and permittivity due to the conductor's presence, it suffices to introduce an equivalent surface current density $\jmath_{s}$ related to the value of the field $E_{z 0}$ on the boundary by means of the differential surface admittance operator $\mathcal{Y}$ defined as

$$
\jmath_{s}=\mathcal{Y} E_{z 0}=\frac{1}{j \omega \mu_{0}}\left[\mathcal{D}_{k}-\mathcal{D}_{k_{\text {out }}}\right] \cdot E_{z 0} .
$$

This is depicted in Fig. 2. The layered medium now permeates the conductor. When solving the field problem external to the conductor, the effect of the conductor is exactly accounted for by the presence of the surface currents $\jmath_{s}$ provided $E_{z 0}$ and the surface current $\jmath_{s}$ are forced to satisfy (9) on $c$. As a matter of fact, by enforcing (9), we ensure that the ratio of the total tangential electric field to the total tangential magnetic field just outside the conductor's surface $S$ is exactly the ratio enforced by the presence of the conductor. This guarantees a unique field solution, notwithstanding possible resonances in $S$. When solving the field problem of Fig. 2, the obtained result is only identical to the one for the original configuration of Fig. 1, taken outside the conductor. Inside the conductor, a fictitious field is obtained. However, in order to obtain relevant data such as total joule losses, total conduction current, or inductance and resistance matrices, the sole knowledge of the surface current density $\jmath_{s}$ suffices. If needed at all, the actual electric field $E_{z}$ inside the conductor can be reconstructed from the knowledge of $E_{z}$ on the boundary $c$. Remark that the surface current does not introduce a discontinuity in the electric field. Hence, we have that on the boundary $c$ and only on $c, E_{z 0}=E_{z}$.

\section{DIRICHLET CHARACTERIZATION}

There are several possibilities to characterize the $\mathcal{Y}$ operator in (9). We briefly come back to this point in Section VII. In the sequel, we opt for a solution based on the Dirichlet eigenfunctions of the cross section $S$. To this end, consider the two Helmholtz Dirichlet problems

$$
\begin{aligned}
\nabla^{2} E_{z}+k^{2} E_{z} & =0 \text { in } S, & & E_{z}=\psi \text { on } c \\
\nabla^{2} E_{z 0}+k_{\text {out }}^{2} E_{z 0} & =0 \text { in } S, & & E_{z 0}=\psi \text { on } c .
\end{aligned}
$$

Putting $\mathcal{E}=E_{z}-E_{z 0}$, we can write

$$
\nabla^{2} \mathcal{E}+k_{\text {out }}^{2} \mathcal{E}=\left(k_{\text {out }}^{2}-k^{2}\right) E_{z}
$$

Since $\mathcal{E}=0$ on $c$, we may expand $\mathcal{E}$ in terms of the orthonormal Dirichlet eigenfunctions $\xi_{m}(\mathbf{r})$ for $S$, which is supposed to form a complete orthonormal basis in $L_{2}(S)$. From (12), we obtain

$$
\mathcal{E}(\mathbf{R})=\left(k_{\text {out }}^{2}-k^{2}\right) \sum_{m=1}^{\infty} \frac{\xi_{m}(\mathbf{R})}{k_{\text {out }}^{2}-\lambda_{m}} \int_{S} \xi_{m}(\mathbf{r}) E_{z}(\mathbf{r}) d S
$$

with $\lambda_{m}$, the eigenvalue corresponding to $\xi_{m}$. Now since $\nabla^{2} \xi_{m}+\lambda_{m} \xi_{m}=0$, it is a simple matter to show that

$$
\int_{S} \xi_{m}(\mathbf{r}) E_{z}(\mathbf{r}) d S=\frac{\oint_{c} \psi(\mathbf{r}) \partial_{n} \xi_{m}(\mathbf{r}) d c}{k^{2}-\lambda_{m}}
$$

resulting in

$$
\mathcal{E}(\mathbf{R})=\left(k_{\text {out }}^{2}-k^{2}\right) \sum_{m=1}^{\infty} \frac{\xi_{m}(\mathbf{R}) \oint_{c} \partial_{n} \xi_{m}(\mathbf{r}) \psi(\mathbf{r}) d c}{\left(k^{2}-\lambda_{m}\right)\left(k_{\text {out }}^{2}-\lambda_{m}\right)}
$$

and, hence, by taking normal derivatives

$$
\begin{aligned}
\jmath_{s}= & \frac{\partial_{n} \mathcal{E}(\mathbf{R})}{j \omega \mu_{0}} \\
= & \frac{k_{\text {out }}^{2}-k^{2}}{j \omega \mu_{0}} \\
& \times \sum_{m=1}^{\infty} \frac{\partial_{n} \xi_{m}(\mathbf{R}) \oint_{c} \partial_{n} \xi_{m}(\mathbf{r}) \psi(\mathbf{r}) d c}{\left(k^{2}-\lambda_{m}\right)\left(k_{\text {out }}^{2}-\lambda_{m}\right)} \quad \mathbf{R} \text { on } c .
\end{aligned}
$$

In shorthand notation, we have

$$
\jmath_{s}=\mathcal{Y} \psi=\tau \sum_{m=1}^{\infty} \frac{\partial_{n} \xi_{m} \oint_{c} \psi \partial_{n} \xi_{m} d c}{\left(k_{\text {out }}^{2}-\lambda_{m}\right)\left(k^{2}-\lambda_{m}\right)}
$$

where the contrast parameter $\tau$ is

$$
\tau=\frac{k_{\mathrm{out}}^{2}-k^{2}}{j \omega \mu_{0}}=\sigma-\sigma_{\mathrm{out}}+j \omega\left(\epsilon-\epsilon_{\mathrm{out}}\right) .
$$

Result (17) is the analytical expression for the differential surface admittance operator $\mathcal{Y}$ operating on the tangential electric 
field $E_{z}=E_{z 0}=\psi$ on $c . \mathcal{Y}$ is expressed in terms of an expansion in the normal derivatives on the boundary $c$ of the Dirichlet eigenfunctions of the conductor's cross section $S$.

\section{A. Joule Losses and Total Surface Current}

The surface joule losses are given by

$$
\mathcal{P}_{s}=\Re\left\{\oint_{c} \jmath_{s} \psi^{*} d c\right\}=\sigma \int_{S}\left|E_{z}\right|^{2} d S-\sigma_{\text {out }} \int_{S}\left|E_{z 0}\right|^{2} d S
$$

which is equal to the difference between the joule volume losses. Relationship (19) follows straightforwardly from Green's identity for functions $E_{z}$ satisfying the Helmholtz equation (10) with a complex wavenumber $k$, namely,

$$
\Im\left\{k^{2}\right\} \int_{S}\left|E_{z}\right|^{2} d S=-\Im\left\{\oint_{c} E_{z}^{*} \partial_{n} E_{z} d c\right\} .
$$

Finally, it should be noted that the total surface current is

$$
\oint_{c} \jmath_{s} d c=(\sigma+j \omega \epsilon) \int_{S} E_{z} d S-\left(\sigma_{\text {out }}+j \omega \epsilon_{\text {out }}\right) \int_{S} E_{z 0} d S
$$

which is equal to the difference between the volume currents (including the displacement currents). Relationship (21) follows straightforwardly from the Gauss divergence theorem applied to Helmholtz equation (10), namely,

$$
\oint_{c} \partial_{n} E_{z} d c=-k^{2} \int_{S} E_{z} d S
$$

and, similarly, for $E_{z 0}$. In the sequel, attention is focused on good conductors embedded in a lossless layer, i.e., $\sigma_{\text {out }}=0$ and $\sigma \gg \omega\left(\epsilon-\epsilon_{\text {out }}\right)$. In that case, the contrast parameter (18) $\tau \approx \sigma$ and the total surface current (21) reduces to the total conduction current in the conductor. The surface joules losses (19) are the total joule losses in the conductor.

\section{B. Pouillet's Law}

In our derivations, $\psi$ represents the electric field $E_{z}$ on the boundary $c$. In the dc case $\omega=0$, we may expect $\psi$ to be constant on $c$ and, as a consequence, we then have that $E_{z}$ is constant over the entire cross section $S$. When $\omega=0$, (17) becomes, with $\psi$ constant and $\sigma_{\text {out }}=0$,

$$
\jmath_{s}=\sigma \psi \sum_{m=1}^{\infty} \frac{\partial_{n} \xi_{m} \oint_{c} \partial_{n} \xi_{m} d c}{\lambda_{m}^{2}} .
$$

Integrating $\jmath_{s}$ over $c$ and using the Gauss identities

$$
\oint_{c} \partial_{n} \xi_{m} d c=-\lambda_{m} \int_{S} \xi_{m} d S
$$

we obtain for the total current

$$
I=\oint_{c} \jmath_{s} d c=\sigma \psi \sum_{m=1}^{\infty}\left(\int_{S} \xi_{m} d S\right)^{2} .
$$

Now the unit function can be developed as

$$
1=\sum_{m=1}^{\infty} \xi_{m}(\mathbf{r}) \int_{S} \xi_{m} d S \quad \mathbf{r} \text { in } S .
$$

By Parseval's identity, we, therefore, obtain

$$
I=(\sigma \psi) S .
$$

Note that this could also have been obtained by exploiting result (21). With $E_{z}=\psi$, this can be written as $R I=E_{z}$, where $R$ is the per unit length resistance $R=(1 / \sigma S)$, which is the well-known Pouillet law.

\section{Example: Circular Region}

The circular region $S$ with radius $r=a$ exhibits very simple formulas. Expanding $E_{z}(a, \theta)=\psi(\theta)$ in a Fourier series as

$$
\psi(\theta)=c_{0}+\sum_{n=1}^{\infty}\left(c_{n} e^{j n \theta}+c_{-n} e^{-j n \theta}\right)
$$

we straightforwardly obtain

$$
E_{z}(r, \theta)=c_{0} \frac{J_{0}(k r)}{J_{0}(k a)}+\sum_{n=1}^{\infty} \frac{J_{n}(k r)}{J_{n}(k a)}\left(c_{n} e^{j n \theta}+c_{-n} e^{-j n \theta}\right)
$$

where $J .(\cdot)$ are the Bessel functions and, hence,

$$
\begin{aligned}
\jmath_{s}(\theta)= & c_{0} \frac{1}{j \omega \mu_{0}}\left[\frac{k J_{0}^{\prime}(k a)}{J_{0}(k a)}-\frac{k_{\text {out }} J_{0}^{\prime}\left(k_{\text {out }} a\right)}{J_{0}\left(k_{\text {out }} a\right)}\right] \\
& +\sum_{n=1}^{\infty} \frac{1}{j \omega \mu_{0}}\left[\frac{k J_{n}^{\prime}(k a)}{J_{n}(k a)}-\frac{k_{\text {out }} J_{n}^{\prime}\left(k_{\text {out }} a\right)}{J_{n}\left(k_{\text {out }} a\right)}\right] \\
& \times\left(c_{n} e^{j n \theta}+c_{-n} e^{-j n \theta}\right) .
\end{aligned}
$$

Note that, in order to obtain (30) for the surface current $\jmath_{s}(\theta)$, we did not, in fact, need the Dirichlet eigenfunctions for the circular region, which is exceptional.

\section{RECTANGULAR REGION}

\section{A. Analytical Solution}

Consider the practically important rectangular region $0 \leq$ $x \leq a$ and $0 \leq y \leq b$. To obtain the differential admittance operator $\mathcal{Y}(17)$, we need the Dirichlet eigenfunctions and eigenvalues of the rectangle, which are

$$
\begin{aligned}
\xi_{m n} & =\frac{2}{\sqrt{a b}} \sin \left(\frac{m \pi x}{a}\right) \sin \left(\frac{n \pi y}{b}\right) \\
\lambda_{m n} & =\left(\frac{m \pi}{a}\right)^{2}+\left(\frac{n \pi}{b}\right)^{2} .
\end{aligned}
$$

The normal derivatives $\partial_{n} \xi_{m n}$ are

$$
\begin{aligned}
& \text { side } 1: y=0: \partial_{n} \xi_{m n}=-\frac{2}{\sqrt{a b}} \frac{n \pi}{b} \sin \left(\frac{m \pi x}{a}\right) \\
& \text { side } 2: x=a: \partial_{n} \xi_{m n}=\frac{2}{\sqrt{a b}} \frac{m \pi}{a}(-1)^{m} \sin \left(\frac{n \pi y}{b}\right)
\end{aligned}
$$




$$
\begin{aligned}
& \text { side } 3: y=b: \partial_{n} \xi_{m n}=\frac{2}{\sqrt{a b}} \frac{n \pi}{b}(-1)^{n} \sin \left(\frac{m \pi x}{a}\right) \\
& \text { side } 4: x=0: \partial_{n} \xi_{m n}=-\frac{2}{\sqrt{a b}} \frac{m \pi}{a} \sin \left(\frac{n \pi y}{b}\right) .
\end{aligned}
$$

It is clear that, on all four sides, the field data preferably have to be developed in a Fourier sine series. Hence, consider $\psi$ on side 1 (the other sides can be treated similarly) given by one sine mode

$$
\psi=\sin \left(\frac{m \pi x}{a}\right)
$$

and $\psi=0$ on the other sides. We readily obtain the surface current density

$$
\jmath_{s}=-\frac{2 \tau b^{2}}{\pi^{3}} \partial_{n}\left\{\sin (m \pi x / a) \Omega\left(\pi y / b, p_{m}, q_{m}\right)\right\}
$$

where

$$
\Omega(u, p, q)=\sum_{j=1}^{\infty} \frac{j \sin (j u)}{\left(p^{2}-j^{2}\right)\left(q^{2}-j^{2}\right)}
$$

and

$$
\begin{aligned}
& p_{m}=\frac{b}{\pi} \sqrt{k^{2}-\left(\frac{m \pi}{a}\right)^{2}} \\
& q_{m}=\frac{b}{\pi} \sqrt{k_{\text {out }}^{2}-\left(\frac{m \pi}{a}\right)^{2}} .
\end{aligned}
$$

Explicit analytic expressions for the function $\Omega(u, p, q)$ and its derivative with respect to $u$ are given in the Appendix. From (37), we easily derive that

$$
\begin{aligned}
& \text { side } 1: y=0: \jmath_{s}=\frac{2 \tau b}{\pi^{2}} \sin (m \pi x / a) \Omega^{\prime}\left(0, p_{m}, q_{m}\right) \\
& \text { side } 2: x=a: \jmath_{s}=-\frac{2 \tau m b^{2}}{\pi^{2} a}(-1)^{m} \Omega\left(\pi y / b, p_{m}, q_{m}\right) \\
& \text { side } 3: y=b: \jmath_{s}=-\frac{2 \tau b}{\pi^{2}} \sin (m \pi x / a) \Omega^{\prime}\left(\pi, p_{m}, q_{m}\right) \\
& \text { side } 4: x=0: \jmath_{s}=\frac{2 \tau m b^{2}}{\pi^{2} a} \Omega\left(\pi y / b, p_{m}, q_{m}\right)
\end{aligned}
$$

where $\Omega^{\prime}$ denotes the derivative of $\Omega(38)$ with respect to its argument $u$.

\section{B. Discretized Form of the Solution}

In Section IV-A, an analytical description of the differential surface admittance operator was obtained. To determine resistance and inductance matrices of multiconductor transmission lines (the topic of Section V), this operator will be combined with an integral-equation approach to solve the exterior field problem. Hence, some form of discretization of the operator is needed. When using an EFIE for the surface current $\jmath_{s}$, it is well known that a pulse basis representation for this surface current suffices for its correct discretization (whereas in the TE case, piecewise linear basis functions would be needed). Moreover, to solve the integral equation, it is advantageous to use a Galerkin weighting procedure in order to assure good convergence and to obtain a symmetric system matrix. Hence, this section is devoted to the derivation of a discretized form of the differential surface admittance, whereby both the surface current $\jmath_{s}$ and the electric field $\psi$ are projected on a pulse basis. Starting with $\psi$, we have that

$$
\psi(x)=\sum_{j=1}^{N} E_{j} t_{j}(x)
$$

where

$$
\begin{aligned}
t_{j}(x) & =1, & & x_{j-1}<x \leq x_{j} \\
& =0, & & \text { elsewhere }
\end{aligned}
$$

and $x_{0}=0, x_{N}=a . N$ is the number of pulse basis functions along each horizontal side of the rectangle. To connect the pulse basis expansion with the sine basis formulation, we first project (44) onto the sine basis in $[0, a]$ by putting

$$
\psi(x) \approx \sum_{m=1}^{M} A_{m} \sin \frac{m \pi x}{a}
$$

and imposing the Galerkin testing procedure

$$
\begin{aligned}
\int_{0}^{a}\left(\sum_{j=1}^{N} E_{j} t_{j}(x)-\sum_{m=1}^{M} A_{m} \sin \frac{m \pi x}{a}\right) \\
\times \sin \frac{m^{\prime} \pi x}{a} d x=0, \quad m^{\prime}=1, \ldots, M .
\end{aligned}
$$

Grouping the coefficients $A_{m}$ and $E_{j}$, respectively, in the column vectors $\mathbf{A}$ and $\mathbf{E}$, we find that

$$
\mathbf{A}=\frac{2}{a} \mathbf{T} \cdot \mathbf{E}
$$

where the entries of the $M \times N$ matrix $\mathbf{T}$ are given by

$$
T_{m, j}=\frac{a}{m \pi}\left[\cos \left(\frac{m \pi x_{j-1}}{a}\right)-\cos \left(\frac{m \pi x_{j}}{a}\right)\right] .
$$

The next step is to project $\jmath_{s}$, as defined in (40)-(43), by a similar Galerkin testing procedure, onto the pulse basis on each side of the rectangle. This means we need to find coefficients $J_{\text {,, }}$ such that on

$$
\begin{aligned}
& \text { side } 1: y=0: \jmath_{s}=\sum_{j=1}^{N} J_{1, j} \frac{t_{j}(x)}{x_{j}-x_{j-1}} \\
& \text { side 2 }: x=a: \jmath_{s}=\sum_{l=1}^{L} J_{2, l} \frac{\hat{t}_{l}(y)}{y_{l}-y_{l-1}} \\
& \text { side } 3: y=b: \jmath_{s}=\sum_{j=1}^{N} J_{3, j} \frac{t_{j}(x)}{x_{j}-x_{j-1}} \\
& \text { side } 4: x=0: \jmath_{s}=\sum_{l=1}^{L} J_{4, l} \frac{\hat{t}_{l}(y)}{y_{l}-y_{l-1}}
\end{aligned}
$$

where

$$
\begin{aligned}
\hat{t}_{l}(y) & =1, & & y_{l-1}<y \leq y_{l} \\
& =0, & & \text { elsewhere }
\end{aligned}
$$


and $y_{0}=0, y_{L}=b$. The surface current representations

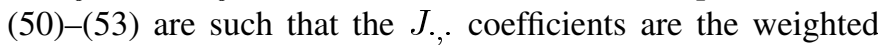
values of $\jmath_{s}$ over each interval, as required by the Galerkin procedure. $L$ is the number of pulse basis functions along each vertical side of the rectangle. Grouping the $J_{\text {., }}$ coefficients into four column vectors, we readily obtain for side 1 that

$$
\mathbf{J}_{1}=\frac{2 \pi}{j \omega \mu_{0} S}\left(\mathbf{T}^{T} \mathbf{D}_{1} \mathbf{T}\right) \cdot \mathbf{E}
$$

where $\mathbf{D}_{1}$ is the $M \times M$ diagonal matrix with elements

$$
D_{1, m m}=-\frac{2}{\pi}\left(p_{m}^{2}-q_{m}^{2}\right) \Omega^{\prime}\left(0, p_{m}, q_{m}\right) .
$$

Similarly, for side 3 , we obtain

$$
\mathbf{J}_{3}=-\frac{2 \pi}{j \omega \mu_{0} S}\left(\mathbf{T}^{T} \mathbf{D}_{3} \mathbf{T}\right) \cdot \mathbf{E}
$$

with the elements of the $M \times M$ diagonal matrix $\mathbf{D}_{3}$ given by

$$
D_{3, m m}=-\frac{2}{\pi}\left(p_{m}^{2}-q_{m}^{2}\right) \Omega^{\prime}\left(\pi, p_{m}, q_{m}\right) .
$$

The results for sides 2 and 4 are less straightforward. After some tedious algebra, we find for side 2 that

$$
\mathbf{J}_{2}=-\frac{4 \tau}{S}\left(\mathbf{U}^{\mathbf{T}} \mathbf{N N}_{\mathbf{n m}} \hat{\mathbf{M T}}\right) \cdot \mathbf{E}
$$

where $\hat{\mathbf{M}}$ is the $M \times M$ diagonal matrix with entries $\hat{M}_{m m}=$ $(-1)^{m}(m \pi / a)$, where $\mathbf{N}$ is the $M \times M$ diagonal matrix with entries $N_{n n}=(n \pi / b)$ and where the entries of the $M \times M$ matrix $\mathbf{N}_{\mathbf{n m}}$ and of the $M \times L$ matrix $\mathbf{U}$ are defined as

$$
\begin{aligned}
N_{n m} & =\frac{1}{\left(k^{2}-\lambda_{n m}\right)\left(k_{\text {out }}^{2}-\lambda_{n m}\right)} \\
U_{n l} & =\frac{b}{n \pi}\left(\cos \frac{n \pi y_{l-1}}{b}-\cos \frac{n \pi y_{l}}{b}\right) .
\end{aligned}
$$

In using the same dimensions for $\hat{\mathbf{M}}$ and $\mathbf{N}$, we have tacitly assumed that the same number of sine functions is used on all sides of the rectangle. Finally, for side 4 , we have

$$
\mathbf{J}_{4}=\frac{4 \tau}{S}\left(\mathbf{U}^{\mathbf{T}} \mathbf{N N}_{\mathrm{nm}} \mathbf{M T}\right) \cdot \mathbf{E}
$$

with the diagonal elements of $\mathbf{M}$ given by $M_{m m}=(m \pi / a)$. This then defines the differential surface admittance operator for the pulse basis excitation on side 1 .

The solution to the complete problem can be obtained by repeating the process for the other three sides and applying superposition. In block diagonal form, the final result can be written as

$$
\left(\begin{array}{l}
\mathbf{J}_{1} \\
\mathbf{J}_{2} \\
\mathbf{J}_{3} \\
\mathbf{J}_{4}
\end{array}\right)=\left(\begin{array}{llll}
\mathbf{J}_{11} & \mathbf{J}_{12} & \mathbf{J}_{13} & \mathbf{J}_{14} \\
\mathbf{J}_{12}^{T} & \mathbf{J}_{22} & \mathbf{J}_{23} & \mathbf{J}_{24} \\
\mathbf{J}_{13}^{T} & \mathbf{J}_{23}^{T} & \mathbf{J}_{11} & \mathbf{J}_{34} \\
\mathbf{J}_{14}^{T} & \mathbf{J}_{24}^{T} & \mathbf{J}_{34}^{T} & \mathbf{J}_{22}
\end{array}\right)\left(\begin{array}{l}
\mathbf{E}_{1} \\
\mathbf{E}_{2} \\
\mathbf{E}_{3} \\
\mathbf{E}_{4}
\end{array}\right)
$$

or, in an easily understood notation,

$$
\mathrm{J}=\mathbf{Y}_{s} \cdot \mathrm{E}
$$

with $\mathbf{E}_{1}=\mathbf{E}$, as introduced above and with $\mathbf{E}_{2}, \mathbf{E}_{3}$, and $\mathbf{E}_{4}$ being the corresponding column vectors formed by the pulse basis expansion coefficients on sides $2-4$. The block matrices in (62) are given by

$$
\begin{aligned}
& \mathbf{J}_{11}=\alpha \mathbf{T}^{T} \mathbf{D}_{1} \mathbf{T}=\mathbf{J}_{11}^{T} \\
& \mathbf{J}_{12}=-\beta \mathbf{T}^{T} \hat{\mathbf{M}} \mathbf{N}_{\mathbf{n m}}^{T} \mathbf{N} \mathbf{U} \\
& \mathbf{J}_{13}=-\alpha \mathbf{T}^{T} \mathbf{D}_{3} \mathbf{T}=\mathbf{J}_{13}^{T} \\
& \mathbf{J}_{14}=\beta \mathbf{T}^{T} \mathbf{M N}_{\mathbf{n m}}^{T} \mathbf{N U} \\
& \mathbf{J}_{22}=\alpha \mathbf{U}^{T} \mathbf{D}_{2} \mathbf{U}=\mathbf{J}_{22}^{T} \\
& \mathbf{J}_{23}=\beta \mathbf{U}^{T} \hat{\mathbf{N}} \mathbf{N}_{\mathbf{n m}}^{T} \hat{\mathbf{M}} \\
& \mathbf{J}_{24}=-\alpha \mathbf{U}^{T} \mathbf{D}_{4} \mathbf{U}=\mathbf{J}_{24}^{T} \\
& \mathbf{J}_{34}=-\beta \mathbf{T}^{T} \mathbf{M} \mathbf{N}_{\mathbf{n m}}^{T} \hat{\mathbf{N U}}
\end{aligned}
$$

with

$$
\begin{aligned}
\beta & =\frac{4 \tau}{S} \\
\alpha & =\frac{2 \pi}{j \omega \mu_{0} S}
\end{aligned}
$$

with the diagonal elements of $\hat{\mathbf{N}}$ given by $(-1)^{n}(n \pi / b)$ and with the elements of $\mathbf{D}_{\mathbf{2}}$ and $\mathbf{D}_{\mathbf{4}}$ defined in the same way as in (56) and (58), but with $m$ replaced by $n$.

$\mathbf{Y}_{s}$ is the differential surface admittance matrix (all entries of $\mathbf{Y}_{s}$ have dimension $\Omega^{-1}$ ). As expected when applying the Galerkin's procedure, a symmetric matrix is obtained. The reader easily verifies from the formulas given above that $\mathbf{Y}_{s}$ is indeed symmetric.

To conclude, an important remark must be added. Note that in (44) and (46), the number of pulse functions $N$ and the number of sine functions $M$ are not identical. The advantage of projecting the pulse basis on the sine basis comes from the fact that the orthogonality of the sine functions on each side of the rectangle can then be exploited in calculating the boundary integral in (17). On the other hand, the sine functions are zero at the corner points and Gibbs's phenomena will occur when representing the nonvanishing surface currents at those corner points. The problem is easily circumvented by choosing $M \gg N$ (e.g., $N=10$ and $M=50-100$ ). This does not impair the (speed of the) method as the number of sine functions does not influence the number of unknowns that will be used to solve the external field problem. $M$ only occurs in the matrix multiplications in (64). The effect of the choice of $M$ will further be discussed in one of the examples of Section VI-A.

\section{DETERMINATION OF RESISTANCE AND INDUCTANCE MATRICES}

Here, the differential surface impedance concept is exploited to determine the resistance and inductance matrices of a set of $N$ parallel conductors extending along the $z$-axis. The way to tackle this problem, using an EFIE approach, has been treated extensively in the literature, see, e.g., [3]-[6]. Following the approach in [4], the relevant EFIE is

$$
E_{z}(\mathbf{r})=-j \omega A_{z}(\mathbf{r})-\frac{\partial V(\mathbf{r})}{\partial z} .
$$

$E_{z}$ is the longitudinal electric field, $A_{z}$ is the vector potential, $V$ is the scalar potential, and $\mathbf{r}=x \mathbf{u}_{x}+y \mathbf{u}_{y}$. The above expression 
will be valid as long as the cross-sectional dimensions remain small with respect to the wavelength such that the transversal currents are negligible. Using the differential surface admittance approach, the conductors can be replaced by equivalent surface currents $J_{s z}$, as explained in Section II. The vector potential of these currents is given by

$$
A_{z}(\mathbf{r})=-\mu_{0} \oint_{c} J_{s z}\left(\mathbf{r}^{\prime}\right) G\left(\mathbf{r}, \mathbf{r}^{\prime}\right) d c\left(\mathbf{r}^{\prime}\right)
$$

The integration runs over the boundaries of the $N$ conductors. Generally speaking, $G\left(\mathbf{r}, \mathbf{r}^{\prime}\right)$ is the Green's function of the medium the conductors are embedded in. Here, we will restrict ourselves to a homogeneous nonmagnetic space or to a half-space bounded by a perfectly electric conducting (PEC) ground plane. In those cases, $G\left(\mathbf{r}, \mathbf{r}^{\prime}\right)$ is given by

$$
\begin{aligned}
G\left(\mathbf{r}, \mathbf{r}^{\prime}\right) & =\frac{1}{2 \pi} \ln \left|\mathbf{r}-\mathbf{r}^{\prime}\right| \quad \text { for infinite space } \\
& =\frac{1}{2 \pi} \ln \frac{\left|\mathbf{r}-\mathbf{r}^{\prime}\right|}{\left|\mathbf{r}-\mathbf{r}^{\prime \prime}\right|} \quad \text { for a half-space }
\end{aligned}
$$

with $\mathbf{r}^{\prime \prime}$ being the image of $\mathbf{r}^{\prime}$ with respect to the PEC ground plane. As $G\left(\mathbf{r}, \mathbf{r}^{\prime}\right)$ must be dimensionless, $1 \mathrm{~m}$ must be supposed in the denominator of the Green's function for infinite space. In solving (66) for good conductors, one supposes that $(\partial V(\mathbf{r}) / \partial z)$ remains constant over the cross section of each conductor. The relationship between these constant values and the total currents through each conductor is

$$
\frac{\partial \mathbf{V}}{\partial z}=-(\mathbf{R}+j \omega \mathrm{L}) \mathbf{I}
$$

In (70), $\mathbf{V}$ is an $N \times 1$ column vector formed by the constant potentials $V_{p}$ of each conductor cross section with $p=$ $1,2, \ldots, N . I$ is also an $N \times 1$ column vector formed by the total currents $I_{p}$ through each conductor $p$ and $\mathrm{R}$ and L, respectively, represent the $N \times N$ resistance and inductance matrix. As a matter of fact, (70) defines the resistance and inductance matrices, i.e., there exists a linear relationship between the total currents flowing through each conductor and the derivatives with respect to the longitudinal coordinate $z$ of the potentials of each of these conductors. In our numerical procedure, the total currents will be judiciously enforced and, from the resulting potentials on each conductor, all elements of the resistance and inductance matrices can then be derived.

We discretize (66) using a point-matching technique and pulse basis functions for the unknown surface currents $J_{s z}$. To this end, the circumferences of the conductors are divided into equal segments, and the surface current takes a constant value on each segment. The fact that we restrict ourselves to equal segments is not due to restrictions imposed by the differential surface admittance formulation. Indeed, result (63) remains valid for arbitrary pulse functions as at the start of the calculations (44), a nonuniform set of pulse functions is assumed. Here, we made the particular choice of uniform sampling to simplify the numerical implementation of the EFIE. In all of the numerical examples, we have taken care to check the convergence of the numerical results for an increasing number of pulses on each side. When trying to restrict the number of pulses per side for a prescribed accuracy, one can, however, expect that, e.g., an edge mesh, as used in the modeling of planar circuits, might be advantageous. This remains to be further investigated.

The total number of segments, taken over all conductor boundaries, is $T$. Taking into account (70) then leads to

$$
E_{i}+j \omega \mu_{0} \sum_{j=1}^{T} G_{i j} J_{j}=\sum_{q=1}^{N}\left(\mathrm{R}_{p q}+j \omega \mathrm{L}_{p q}\right) I_{q}
$$

$E_{i}$ represents the value of the electric field at the center of segment $i$ and $i=1,2, \ldots, T . G_{i j}$ results from the discretization of the boundary integrals in (67) and expresses the interaction between segment $i$ and segment $j$ (remark that $G_{i j}=G_{j i}$ ). Further suppose that segment $i$ is located on conductor $p$. In the right-hand-side member of (71), the summation runs over all conductors and involves the self-coupling and mutual-coupling resistances and inductances between conductor $p$ and all other conductors. Also remark that the resistance and inductance matrix are symmetrical and, hence, $\mathrm{R}_{p q}=\mathrm{R}_{q p}$ and $\mathrm{L}_{p q}=\mathrm{L}_{q p}$. To simplify the further discussion, we finally collect all $E_{i}$ 's into a $T \times 1$ column vector $\mathrm{E}$ and all $J_{j}$ 's into a $T \times 1$ column vector J. The $G_{i j}$ 's are collected in a $T \times T$ matrix $\mathrm{G}$. The right-hand-side members of (71) can be concisely written as a $T \times 1$ column vector $U$ whereby element $U_{i}$ is identical for all segments $i$ on the same conductor. With the above definitions, the set of equations that govern the problem can be compactly represented as

$$
\mathrm{E}+j \omega \mu_{0} \mathrm{G} \cdot \mathrm{J}=\mathrm{U}
$$

To take into account (63), relating all electric fields on the same conductor to the equivalent surface currents on the boundary of that conductor, a block-diagonal total differential surface admittance matrix $Y_{\text {tot }}$ is formed as follows:

$$
\mathbf{Y}_{\mathrm{tot}}=\left[\begin{array}{ccc}
\mathbf{Y}_{s 1} & 0 & \cdots \\
0 & \mathbf{Y}_{s 2} & \ldots \\
\cdots & \cdots & \ldots \\
\cdots & 0 & \mathbf{Y}_{s N}
\end{array}\right]
$$

with $\mathbf{Y}_{s p}$ being the differential surface admittance matrix for conductor $p$. The final result is now obtained by left-multiplying (72) with $Y_{\text {tot }}$, i.e.,

$$
\mathrm{J}+j \omega \mu_{0}\left(\mathrm{Y}_{\text {tot }} \cdot \mathrm{G}\right) \cdot \mathrm{J}=\mathrm{Y}_{\text {tot }} \cdot \mathrm{U}
$$

The values of the elements of the resistance and inductance matrices can now be obtained by solving (74) $N$ times, enforcing the fact that, for each of these solutions, the total current running through one of the conductors is equal to unity, while all other total currents remain zero. As $Y_{\text {tot }}$ and $G$ are symmetrical, the symmetry of $R$ and $L$ is guaranteed. 


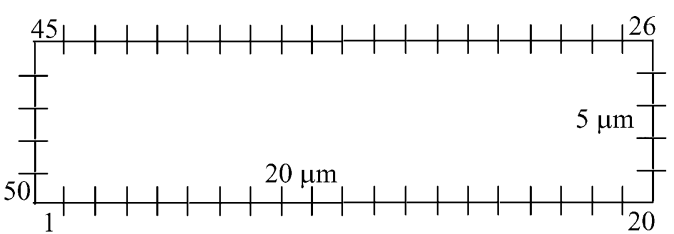

(a)

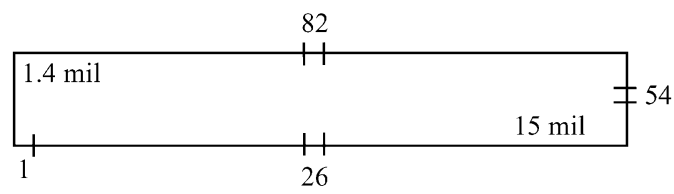

(b)

Fig. 3. (a) $20 \mu \mathrm{m} \times 5 \mu \mathrm{m}$ copper conductor $\left.\left(\sigma=5.8 \times 10^{7}(\Omega \mathrm{m})^{-1}\right)\right)$ (test example also used in [6]). (b) $15 \mathrm{mil} \times 1.4$ mil copper conductor $(\sigma=$ $\left.5.8 \times 10^{7}(\Omega \mathrm{m})^{-1}\right)$ ) (test example also used in [5]).

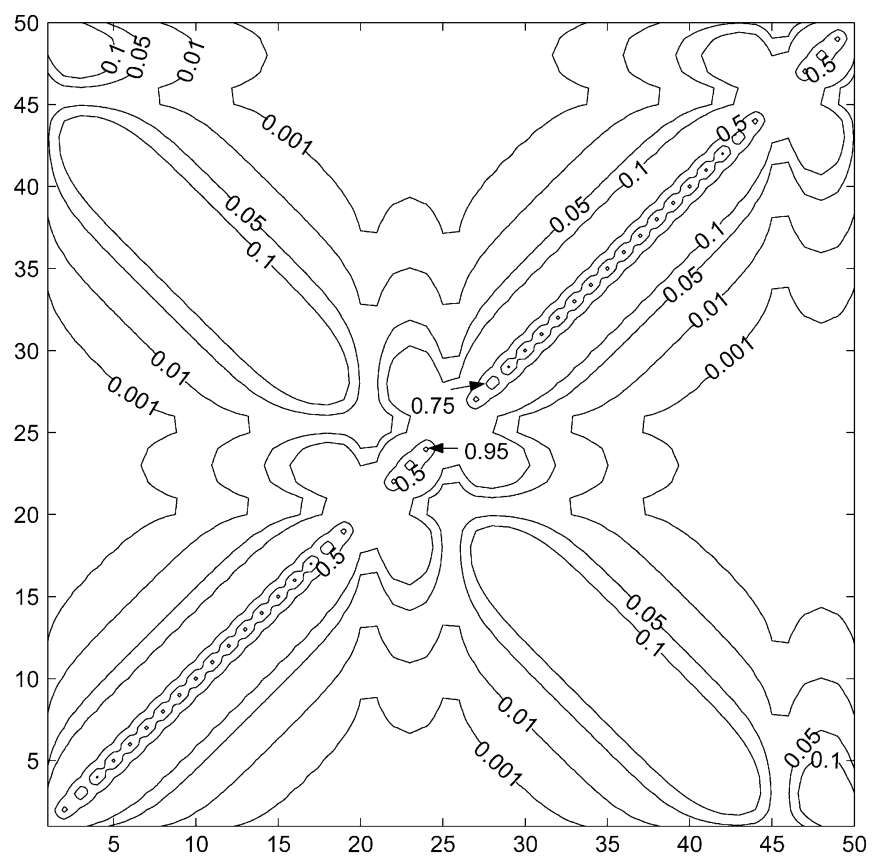

Fig. 4. Contour plot of the normalized absolute value of the elements of the $\mathbf{Y}_{S}$ differential surface admittance matrix of the conductor of Fig. 3(a) at 79.1 MHz.

\section{NUMERICAL RESULTS}

\section{A. Differential Surface Admittance Matrix}

A first set of results is intended to provide the reader with some insight into the behavior of the differential surface admittance matrix $\mathbf{Y}_{s}(63)$. Consider a copper conductor $(\sigma=$ $\left.\left.5.8 \times 10^{7}(\Omega \mathrm{m})^{-1}\right)\right)$ measuring $20 \mu \mathrm{m} \times 5 \mu \mathrm{m}$ [see Fig. 3(a)]. The circumference is subdivided into $N=20$ intervals along the length and $L=5$ intervals along the width, i.e., a total of 50 intervals. The number of sine functions used on each side is $M=100$. A similar example is put forward in [6]. Fig. 4 shows a contour plot of the normalized absolute value of the elements of the $50 \times 50 \mathbf{Y}_{s}$ matrix at $79.1 \mathrm{MHz}$. The numbering on the axes corresponds to the numbering shown on Fig. 3(a) and the plotted values have been normalized with respect to the absolute value of the largest element in the matrix $\left(2.7315 \times 10^{-5}\right)$. This numbering implies that the $\left|\mathbf{Y}_{s}(1,1)\right|$ value is displayed in the lower left-hand-side corner of the figure

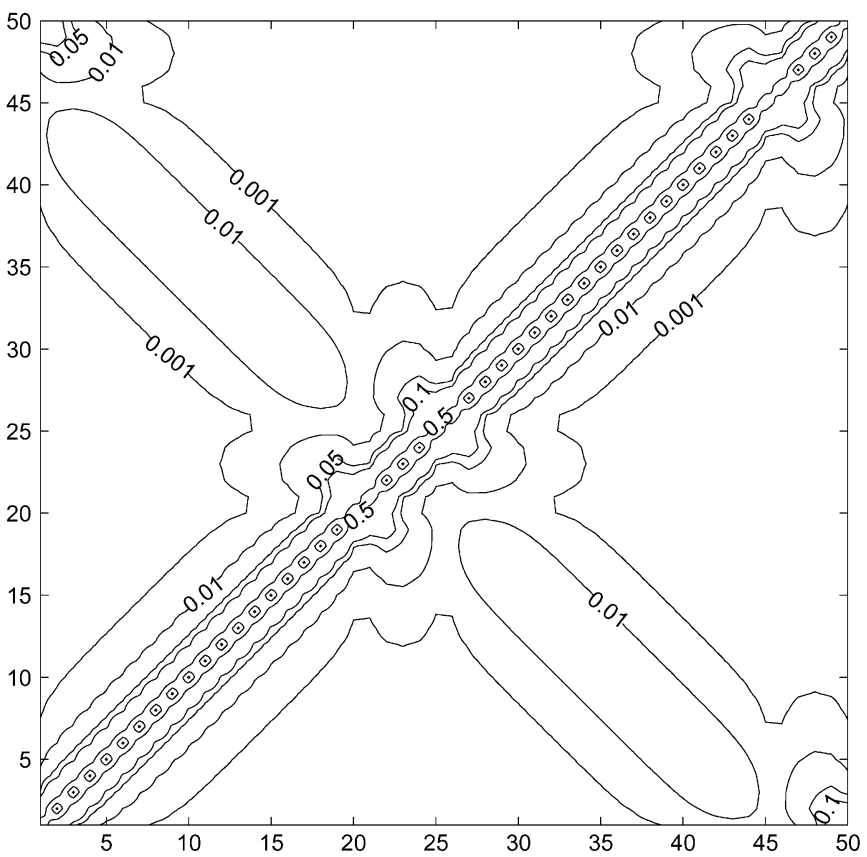

Fig. 5. Contour plot of the normalized absolute value of the elements of the $\mathbf{Y}_{s}$ differential surface admittance matrix of the conductor of Fig. 3(a) at $10 \mathrm{GHz}$.

and the $\left|\mathbf{Y}_{s}(50,50)\right|$ value in the upper right-hand-side corner. Hence, the highest impedance values are to be found along the main diagonal running from the lower left-hand-side corner to the upper right-hand-side corner. The skin depth $\delta=7.43 \mu \mathrm{m}$. Fig. 5 shows the corresponding result at $10 \mathrm{GHz}(\delta=0.66 \mu \mathrm{m}$, maximum value: $\left.1.4390 \times 10^{-5}\right)$. One clearly observes a similar behavior, as reported in [6], for the global surface impedance. The off-diagonal elements quickly decrease with increasing frequency and the influence of the corners is clearly visible. To provide some further quantitative data as a function of frequency, we next consider the $15 \mathrm{mil}(381 \mu \mathrm{m}) \times 1.4 \mathrm{mil}(35.56 \mu \mathrm{m})$ copper conductor $\left.\left(\sigma=5.8 \times 10^{7}(\Omega \mathrm{m})^{-1}\right)\right)$ example discussed in [5] [see Fig. 3(b)]. The circumference is now subdivided into $N=51$ and $L=5$ intervals, i.e., a total of 112 intervals and $M=204$. The chosen value of $M$ will be further discussed below. The solid lines in Fig. 6 show the real part of the following elements of the $\mathbf{Y}_{s}$-matrix: $Y_{26,26}, Y_{54,54}, Y_{26,82}$, and $Y_{1,1}$. As can be seen in Fig. 3(b), interval 26 is located in the middle of the bottom side, interval 54 in the middle of the right-hand side, and interval 82 in the middle of the top side. The $(1,1)$-element has been selected to illustrate the behavior near a corner. Results have been plotted as a function of the skin depth $\delta=\sqrt{\left(2 / \omega \mu_{0} \sigma\right)}$ and the frequency ranges between $0.1 \mathrm{MHz}$ $(\delta=209.98 \mu \mathrm{m})$ and $100 \mathrm{GHz}(\delta=0.209 \mu \mathrm{m})$. The plotted values are normalized with respect to the value of the real part of $Y_{26,26}$ at $0.1 \mathrm{MHz}$, i.e., $1.5 \times 10^{-3}$. The dashed lines show the corresponding results for the absolute value of the imaginary parts. The numerical results clearly show that the real parts are dominant and almost constant when the skin depth is large with respect to the width of the conductor. For increasing frequencies, the imaginary parts gain in importance. When the skin depth becomes very small, all nondiagonal elements have died out and the absolute value of the real and imaginary parts of the diagonal elements become identical. This behavior cannot only 


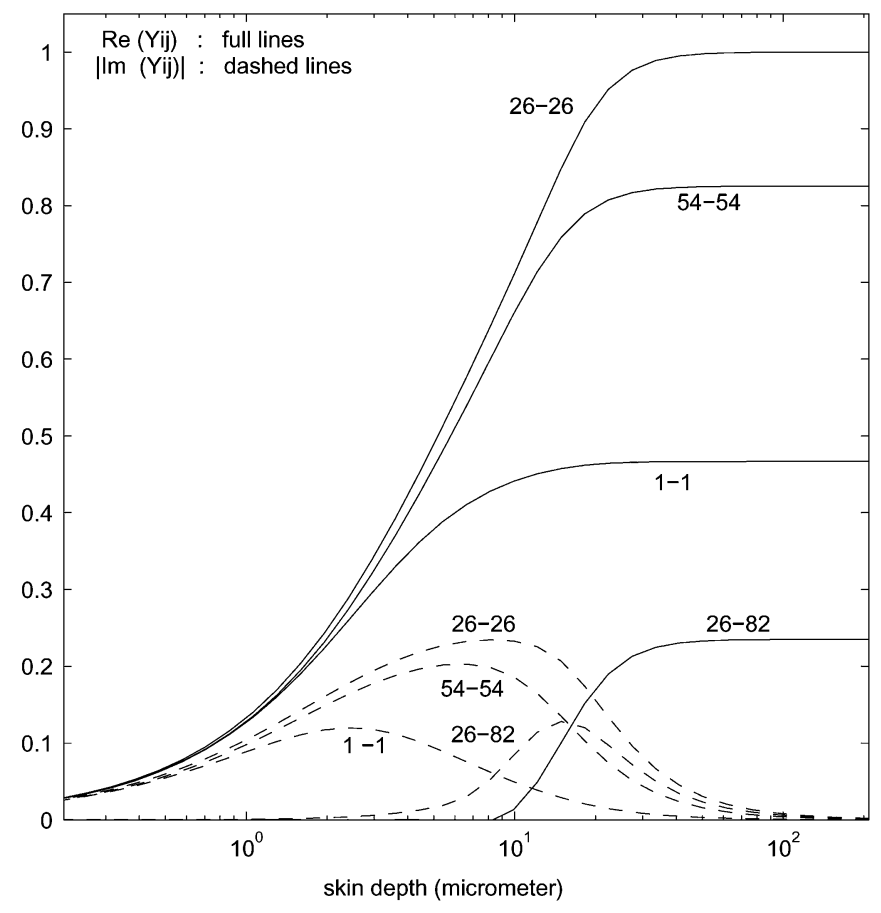

Fig. 6. Normalized value of the real parts of the $\left(\mathbf{Y}_{s}\right)_{i j}$ elements (solid line) and absolute value of the imaginary parts of the $\left(\mathbf{Y}_{s}\right)_{i j}$ elements (dashed line) of the differential surface admittance matrix as a function of skin depth for the conductor of Fig. 3(b).

be verified numerically, but as a matter of fact, follows directly from the high-frequency limit of the coefficient of $\sin (m \pi x / a)$ in (40), namely,

$$
\frac{2 \tau b}{\pi^{2}} \Omega^{\prime}\left(0, p_{m}, q_{m}\right)=\sqrt{\frac{\sigma}{j \omega \mu_{0}}}
$$

for $\omega \rightarrow \infty$. This limiting value is the well-known scalar surface admittance value. In [5], the behavior of the internal impedance of a good conductor $Z_{i}=r_{i}+j \omega l_{i}$ as a function of frequency is examined. For the $15 \times 1.4$ mil example, the authors observe a difference of $20 \%$ between the per-unit-length internal inductive reactance $\omega l_{i}$ and the per-unit-length resistance $r_{i}$ when the skin effect is well developed. At the highest frequency considered by Antonini et al. [5], i.e., $1 \mathrm{GHz}$ or $\delta=2.09 \mu \mathrm{m}$, Fig. 6 shows a difference of approximately $40 \%$ between the real and imaginary parts of the diagonal matrix elements located in the middle of the sides, even increasing to approximately $65 \%$ for the $(1,1)$ matrix element. Although it is clear that the internal impedance considered in [5] and the differential surface admittance considered here are different quantities, our results confirm the observations put forward in [5]. Furthermore, by still increasing the frequency, i.e., for sufficiently small skin depths, the differential surface admittance matrix becomes purely diagonal with all diagonal elements given by (75). This seems to imply that the internal inductive reactance and internal resistance, as considered in [5], will also become identical provided the skin depth is small enough.

Before turning to the calculation of the resistance and inductance matrices of some sample configurations, we would like to illustrate the effect of the number $M$ of sine functions used

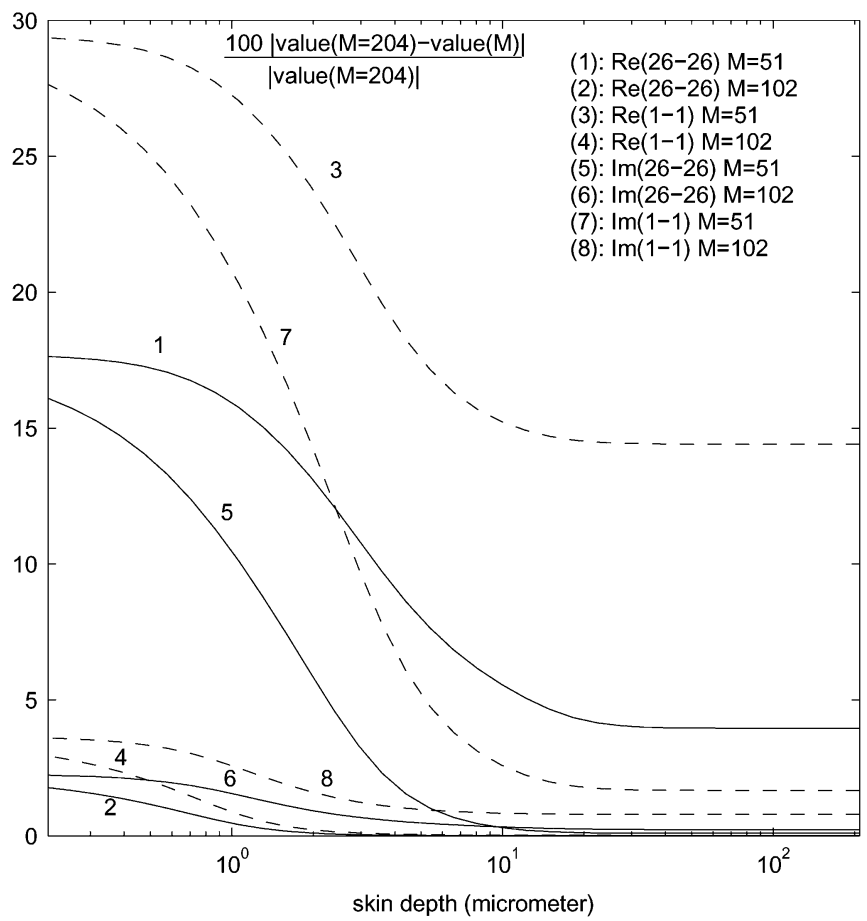

Fig. 7. Illustration of the influence of the number $M$ of sine functions used in the analytical treatment of the rectangular conductor for the case considered in Fig. 6 . The displayed percentages are defined with respect to the values obtained for $M=204$.

in the procedure outlined in Section IV-B. To this end, consider the $(26,26)$ and $(1,1)$ elements already discussed above. These elements are now calculated for three different $M$-values, i.e., $M=51$ (i.e., exactly the number of intervals used on the top and bottom side), $M=102$, and $M=204$. The result for $M=204$ is taken as the reference result as the absolute change in the obtained values is negligible for larger $M$. Fig. 7 shows the relative differences between this reference result and the results for $M=51$ and $M=102$ (i.e., $100|\operatorname{value}(M=204)-\operatorname{value}(M)| /|\operatorname{value}(M=204)|)$ and this, again, as a function of the skin depth. As already remarked at the end of Section IV-B, choosing $M$ sufficiently larger than the number of divisions per side is essential in order to obtain correct results, especially at the higher frequencies and for the corner elements.

\section{B. Resistance and Inductance Matrices}

As our first example, we revisit the isolated square copper conductor $\left.\left(\sigma=5.72 \times 10^{7}(\Omega \mathrm{m})^{-1}\right)\right)$ with side $4.62 \mathrm{~mm}$ also treated in [3]. Fig. 8 shows its resistance in $m \Omega / m$ as a function of frequency. On the scale of this figure, this result almost completely coincides with the hybrid technique (solid line) result calculated and displayed in [3]. In our case, this result was obtained using $N=L=20$ uniformly spaced divisions per side and $M=200$. This particular example has been selected to address the following concern raised by the reviewers. In a surface integral-equation approach of the kind presented here, it could perhaps be expected that a discretization step of, at most, half the skin depth is necessary to obtain correct results. As will be evident from the results given below, this does not really seem to be the case. We believe that the reason for this is the following. In 


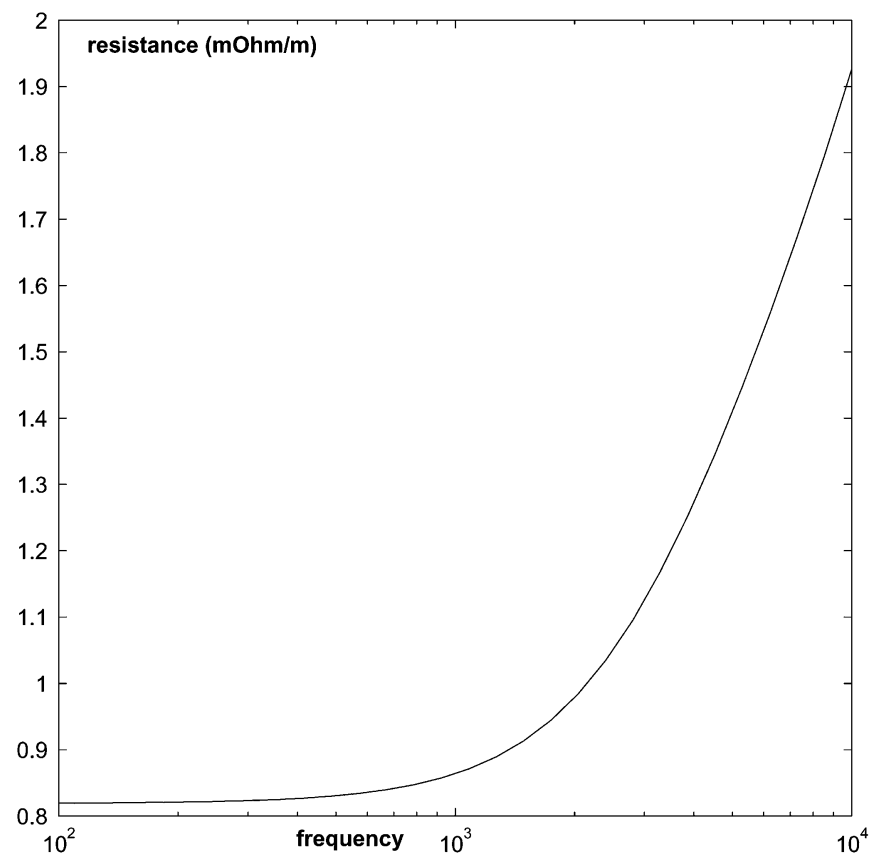

Fig. 8. Resistance in $m \Omega / \mathrm{m}$ as a function of frequency for an isolated square copper conductor $\left(\sigma=5.72 \times 10^{7}(\Omega \mathrm{m})^{-1}\right)$ ) with side $4.62 \mathrm{~mm}$ (example also used in [3]).

TABLE I

RESISTANCE VALUES IN $m \Omega / \mathrm{m}$ AS A FUNCTION OF FREQUENCY FOR THE SQUARE, 4.62-mm SIDE, COPPER $\left.\left(\sigma=5.72 \times 10^{7}(\Omega \mathrm{m})^{-1}\right)\right)$ CONDUCTOR ALSO CONSIDERED IN FIG. 8 AND FOR FOUR DIFFERENT DisCRETIZATIONS

\begin{tabular}{c|c|c|c|c}
\hline frequency $(\mathrm{Hz})$ & $150 \times 150$ & $50 \times 50$ & $20 \times 20$ & $10 \times 10$ \\
\hline $10^{2}$ & 0.8195 & 0.8195 & 0.8196 & 0.8196 \\
$10^{3}$ & 0.8638 & 0.8638 & 0.8637 & 0.8635 \\
$10^{4}$ & 1.927 & 1.927 & 1.926 & 1.920 \\
$10^{5}$ & 5.682 & 5.684 & 5.650 & 5.468 \\
$10^{6}$ & 17.38 & 17.28 & 17.01 & 16.36 \\
\hline
\end{tabular}

a coupled integral-equation approach, such as in [3], which uses both the Green's function of the conductor and the Green's function of the surrounding medium or in a volume integral equation approach, discretization of the order of the skin depth is necessary to capture the current crowding. In our approach, we believe this current crowding is already captured in the differential surface admittance provided enough sine functions are used. Of course, the equivalent surface current exhibits the typical behavior at corner points, i.e., this current will be higher at the corners and, hence, a sufficient discretization is needed to capture this effect, e.g., as is the case for a perfect conductor. Hence, the typical discretization needed in our approach is mainly dominated by the wavelength in the surrounding medium and by the need to capture corner effects. To further illustrate this, Table I gives some numerical data for the example of Fig. 8 for different uniform discretizations $N=L=150, N=L=50, N=$ $L=20$, and $N=L=10$ (for $M=300$ ). The skin depth at the highest frequency is $66.5 \mu \mathrm{m}$. For $N=L=150$, each interval is $30.8 \mu \mathrm{m}$, i.e., about half the skin depth at the highest frequency. From this table, it is clear that the $10 \times 10$ discretization indeed underestimates the losses and that, for the $20 \times 20$

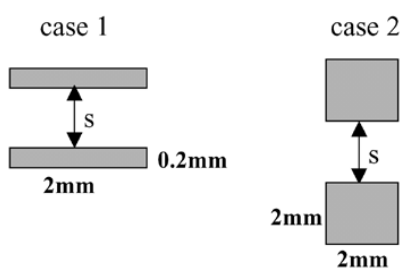

Fig. 9. Two pairs of copper signal conductors $(20 \mathrm{~mm} \times 0.2 \mathrm{~mm}$ and $2 \mathrm{~mm} \times$ $\left.2 \mathrm{~mm}, \sigma=5.6 \times 10^{7}(\Omega \mathrm{m})^{-1}\right)$ ) with variable separation distance $s$ (examples also used in [3] and [6]).

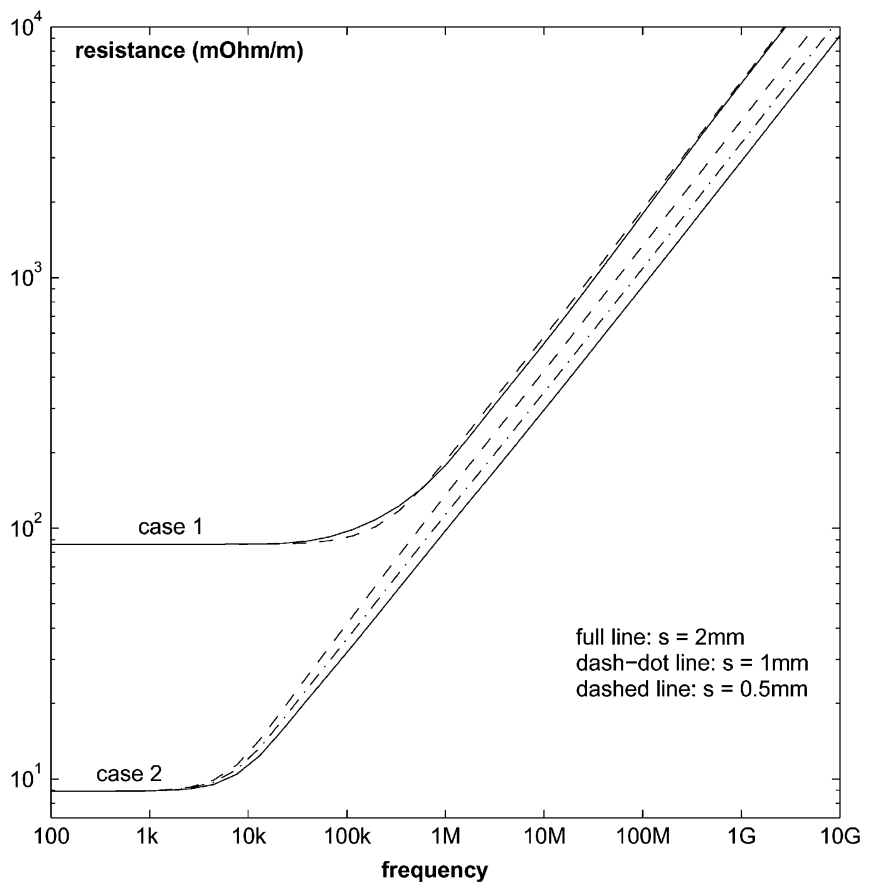

Fig. 10. Resistance in $m \Omega / \mathrm{m}$ as a function of frequency for the configurations of Fig. 9 and for different separations $s$.

discretization, the error is already small. As remarked by the reviewers, a nonuniform discretization will further enhance the convergence of the results. This will be investigated in the future.

As a second example, we consider the copper two-conductor system $\left.\left(\sigma=5.6 \times 10^{7}(\Omega \mathrm{m})^{-1}\right)\right)$ depicted in Fig. 9, treated in [3] and [6]. We considered both the $2 \mathrm{~mm} \times 0.2 \mathrm{~mm}$ case (case 1) and the $2 \mathrm{~mm} \times 2 \mathrm{~mm}$ case (case 2) for separation distances $s=0.5 \mathrm{~mm}, 1 \mathrm{~mm}$, and $2 \mathrm{~mm}$. The number of divisions was 20 on each side $(N=L=20)$ and $M=200$. Fig. 10 shows the resistance results as a function of frequency between $100 \mathrm{~Hz}-10 \mathrm{GHz}$. The dc value is, of course, independent of the separation distance. For case 2, the resistance curves start to differ at a lower frequency as compared to case 1 and the difference between the curves for increasing separation distances is much more important. For case 1, the resistance curves remain very close to each other. For clarity, we have left out the $s=1 \mathrm{~mm}$ result for case 1 . One can also observe that, for case 1 , the curves cross each other near $1 \mathrm{MHz}$. We have carefully verified the influence of changing different parameters $(N, L, M)$, confirming that the observed phenomenon is not an artifact of the calculations. For increasing values of $N$, the resistance values increase by a small percentage (in an analogous 


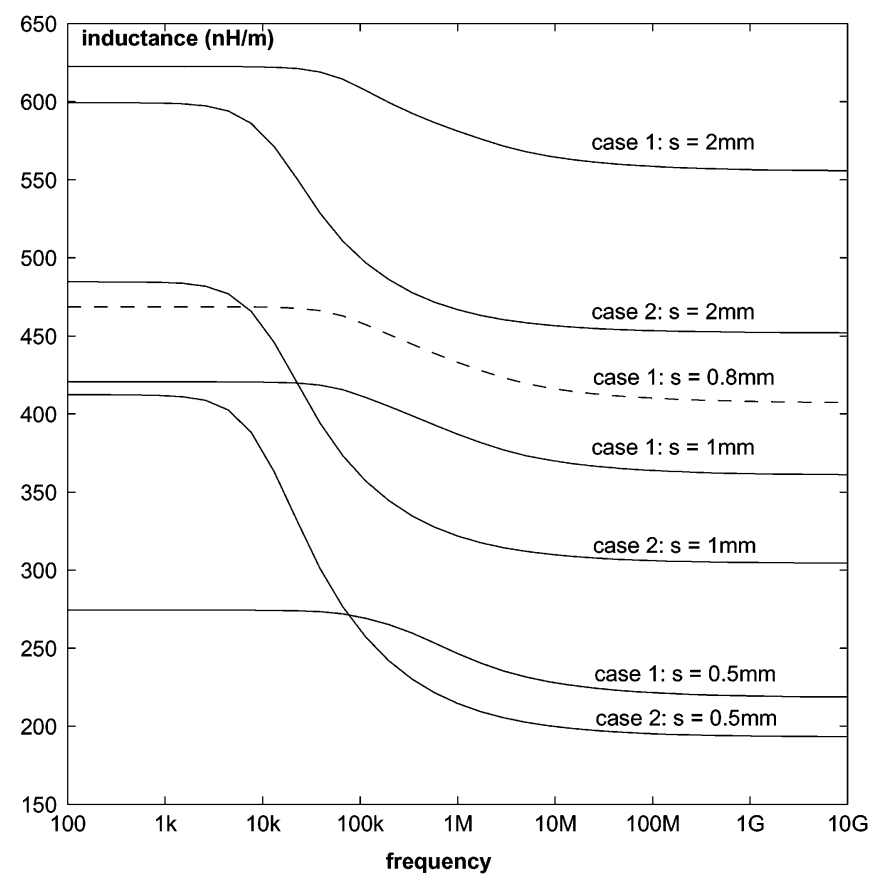

Fig. 11. Inductance in nanohenrys/meter as a function of frequency for the configurations of Fig. 9 and for different separations $s$.

way as denoted by the results in Table I). In Fig. 10, this is hardly visible and the crossing of the curves still occurs at (almost) the same frequency. Fig. 11 shows the corresponding results for the inductance. Observe that the inductance results are much more sensitive to the distance between the conductors. For case 1, the separation distance used in [3] is $s=0.8 \mathrm{~mm}$. The result for this distance is also shown on the plot (dashed line). Using the surface integral-equation approach presented in [10] for PEC multiconductor lines, we could verify that the high-frequency inductance results in Fig. 11 coincide with the results obtained for perfect conductors, as expected. To conclude the discussion of this first example, the reader will remark that our results coincide with those (case 1-0.8 mm and case 2-2 $\mathrm{mm}$ ) presented in [10], but that the low-frequency values in [6] for case 1-1 mm and case 2-2 seem to underestimate the inductance. As pointed out by a reviewer, this is due to the fact that, in [6], a zero tangential electric field has been assumed at the conductor's surface, while this is avoided by the differential admittance procedure only imposing that $E_{z}=E_{z 0}$ on $c$. This leads to an underestimation of the contribution of the internal inductance to the overall inductance and this contribution is more important at the lower frequencies.

Next consider the four conductor (Al-oxide, $\sigma=$ $\left.(1 / 2.8) \times 10^{8}(\Omega \mathrm{m})^{-1}\right)$ configuration depicted in Fig. 12. All dimensions are in micrometers and the boundaries were discretized using ten divisions per micrometer. We first calculated the $4 \times 4$ resistance and inductance matrix and then selected the large "ground" conductor 4 to be the reference conductor (zero potential and $I_{4}=-\left(I_{1}+I_{2}+I_{3}\right)$ ). The resulting configuration is characterized by a $3 \times 3$ resistance and inductance matrix. Fig. 13 shows the resistance elements $R_{11}=R_{33}, R_{22}, R_{12}=R_{23}$, and $R_{13}$ (in $\Omega \mathrm{m}^{-1}$ ) as a function of frequency between $10 \mathrm{MHz}-1 \mathrm{THz}$. The corresponding

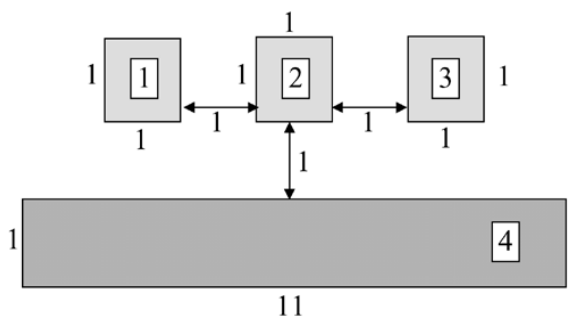

Fig. 12. Three signal and reference Al-oxide $\left.\left(\sigma=1 / 2.8 \times 10^{8}(\Omega \mathrm{m})^{-1}\right)\right)$ conductor configuration (all dimensions are in micrometers).

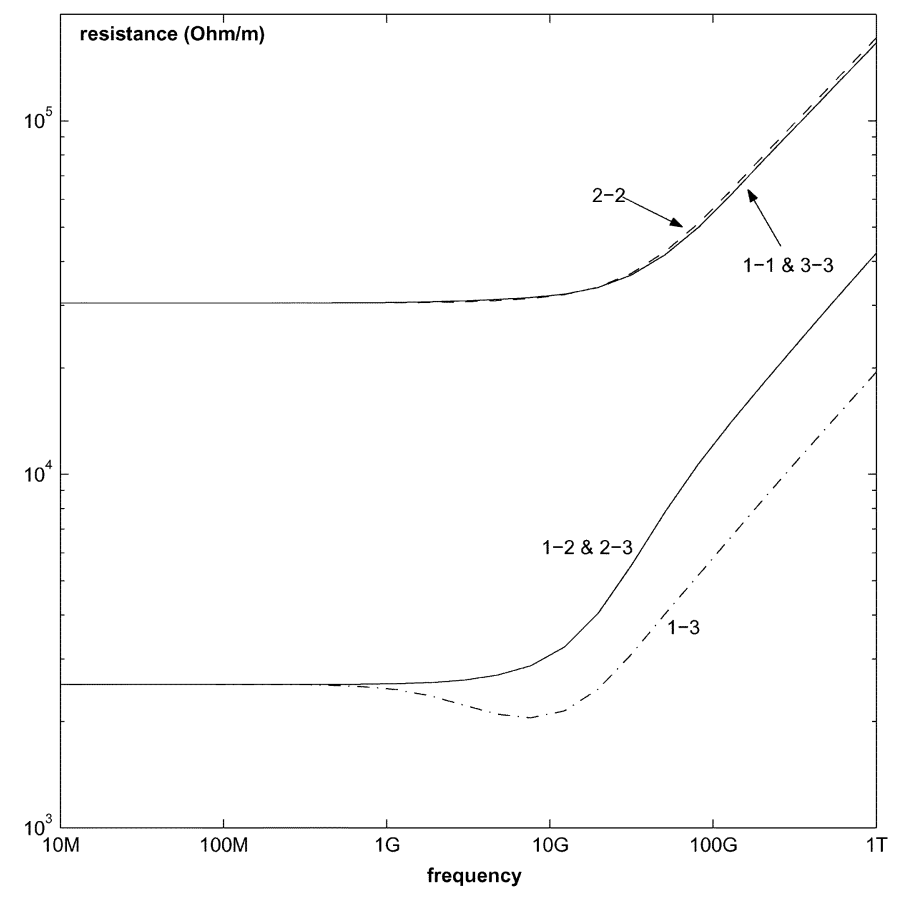

Fig. 13. Resistance matrix elements in $\Omega / \mathrm{m}$ as a function of frequency for the configuration of Fig. 12 and with conductor 4 as the reference conductor.

elements of the inductance matrix $L_{11}=L_{33}, L_{22}, L_{12}=L_{23}$, and $L_{13}\left(\right.$ in $\left.\mathrm{nH} \cdot \mathrm{m}^{-1}\right)$ are displayed in Fig. 14.

The configuration of the last example is depicted in Fig. 15. It is the cross section of a coaxial line with two copper signal conductors surrounded by a copper outer conductor $(\sigma=5.8 \times$ $\left.10^{7}(\Omega \mathrm{m})^{-1}\right)$ ). All dimensions are in units of $0.1 \mathrm{~mm}$ and a discretization of four divisions per $0.1 \mathrm{~mm}$ is used. As our method can only handle rectangular conductors, the outer conductor is subdivided into four separate conductors, as indicated in Fig. 15. However, the gap between the conductors is kept extremely small (less than one-tenth of a micrometer, and we verified numerically that, for such small gaps, stable numerical results are obtained). The total number of segments amounts to 528. Similarly, as for the previous example, we now assign a zero reference potential to the four conductors forming the outer coaxial shield and determine the $2 \times 2$ resistance and inductance matrix of the resulting configuration. The final results are displayed in Fig. 16. The dc value for $R_{11}=R_{22}$ is $1.73408 \Omega / \mathrm{m}$, i.e., the sum of the dc resistance of the outer coaxial shield and one of the signal conductors. The dc value for $R_{12}=R_{21}$ is $2.9829 \times 10^{-2} \Omega / \mathrm{m}$, i.e., the dc resistance of the outer coaxial shield. We further remark that the low-frequency value of $L_{12}=$ 


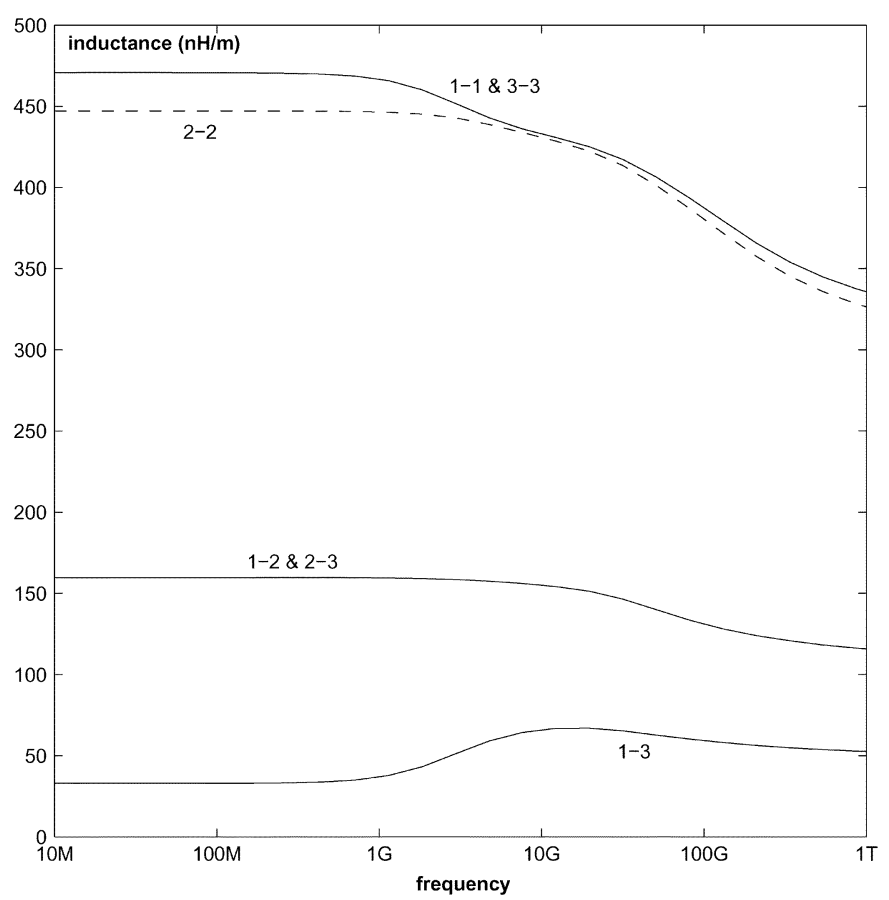

Fig. 14. Inductance matrix elements in nanohenrys per meter as a function of frequency for the configuration of Fig. 12 and with conductor 4 as the reference conductor.

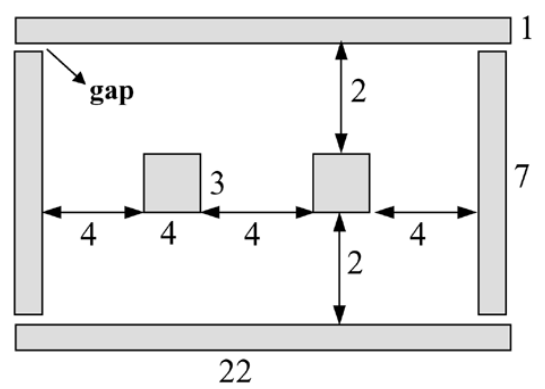

Fig. 15. Cross section of a copper $\left.\left(\sigma=5.8 \times 10^{7}(\Omega \mathrm{m})^{-1}\right)\right)$ coaxial line with two signal conductors (all dimensions are in units of $0.1 \mathrm{~mm}$ ).

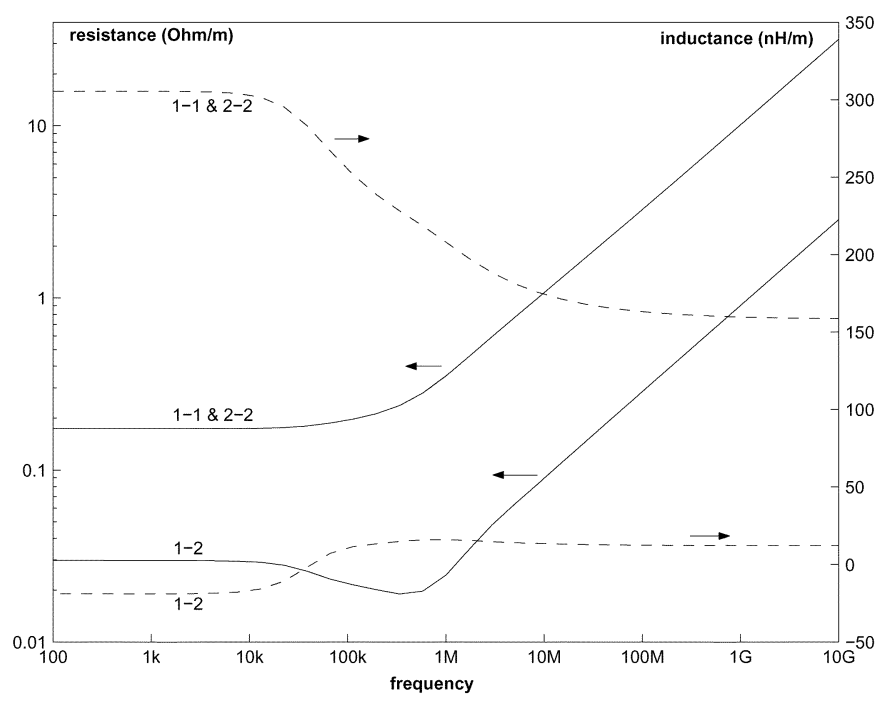

Fig. 16. Resistance (solid line: $\Omega / \mathrm{m}$ ) and inductance (dashed line: nanohenrys per meter) matrix elements as a function of frequency for the configuration of Fig. 15 and with the outer conductor as the reference conductor.
$L_{21}$ is negative. This is allowed as long as the $2 \times 2$ inductance matrix remains positive-definite. This positive-definite nature has been verified for the complete frequency range and implies that the magnetic energy always remains positive whatever the currents used to excite the configuration. We have also determined the inductance matrix for the case of perfect conductors using a finite-difference technique to solve Laplace's equation for the capacitance problem and using the fact that the product of the inductance and capacitance matrix equals $\left(1 / \epsilon_{0} \mu_{0}\right)$. The obtained results are $L_{11}=157.4 \mathrm{nH} / \mathrm{m}$ and $L_{12}=12.06 \mathrm{nH} / \mathrm{m}$, while the present technique yields $L_{11}=158.62 \mathrm{nH} / \mathrm{m}$ and $L_{12}=12.11 \mathrm{nH} / \mathrm{m}$.

\section{CONCLUSION}

In this paper, we have presented the differential surface admittance operator concept for the 2-D TM case. One way to obtain this operator is to use the Dirichlet eigenfunctions of the conductor's cross section, as explained in Section III. This approach is ideally suited for the circular and rectangular cylinder case, as the Dirichlet eigenfunctions are readily available. Another approach, which will be examined in the future, is to derive the admittance operator using an integral-equation approach. The advantage of the latter approach is that general cross sections can be handled more easily. We have explicitly shown that the differential surface admittance operator leads to a correct description of the behavior of the conductor from dc to very high frequencies. Furthermore, it has been demonstrated how the differential surface admittance description can be combined with an EFIE to obtain the resistance and inductance matrices of a set of multiconductor lines. Further research also remains necessary to assess the effect of a nonuniform discretization of the surface current.

\section{APPENDIX}

We need expressions for $\Omega(u, p, q), \Omega^{\prime}(0, p, q)$, and $\Omega^{\prime}(\pi, p, q)$. For $p \neq q \neq 0$, we have [11, Table A.2]

$$
\begin{aligned}
\Omega(u, p, q)=- & \frac{\pi}{2}\left[\frac{\sin (\pi p-p u)}{\left(q^{2}-p^{2}\right) \sin (\pi p)}\right. \\
& \left.+\frac{\sin (\pi q-q u)}{\left(p^{2}-q^{2}\right) \sin (\pi q)}\right], \quad 0 \leq u<2 \pi
\end{aligned}
$$

and for $q=p \neq 0$,

$\Omega(u, p, p)=\frac{\pi}{4} \frac{(\pi-u) \cos (\pi p-p u)-\pi \cot (\pi p) \sin (\pi p-p u)}{p \sin (\pi p)}$.

For $q=0, p \neq 0$, we have

$$
\Omega(u, p, 0)=\frac{(u-\pi) \sin (\pi p)+\pi \sin (\pi p-p u)}{2 p^{2} \sin (\pi p)}
$$

and for $q=p=0$,

$$
\Omega(u, 0,0)=\frac{\pi^{2} u}{6}-\frac{\pi u^{2}}{4}+\frac{u^{3}}{12} .
$$


Furthermore, we have for $p \neq q \neq 0$,

$$
\begin{aligned}
& \Omega^{\prime}(0, p, q)=-\frac{\pi}{2}\left[\frac{p \cot (\pi p)-q \cot (\pi q)}{p^{2}-q^{2}}\right] \\
& \Omega^{\prime}(\pi, p, q)=-\frac{\pi}{2}\left[\frac{p \csc (\pi p)-q \csc (\pi q)}{p^{2}-q^{2}}\right]
\end{aligned}
$$

and for $q=p \neq 0$,

$$
\begin{aligned}
& \Omega^{\prime}(0, p, p)=-\frac{\pi}{2}\left[\frac{\cot (\pi p)-\pi p \csc (\pi p)^{2}}{2 p}\right] \\
& \Omega^{\prime}(\pi, p, p)=-\frac{\pi}{2}\left[\frac{\csc (\pi p)-\pi p \cot (\pi p) \csc (\pi p)}{2 p}\right] .
\end{aligned}
$$

For $q=0, p \neq 0$, we have

$$
\begin{aligned}
& \Omega^{\prime}(0, p, 0)=-\frac{\pi}{2}\left[\frac{p \cot (\pi p)-1 / \pi}{p^{2}}\right] \\
& \Omega^{\prime}(\pi, p, 0)=-\frac{\pi}{2}\left[\frac{p \csc (\pi p)-1 / \pi}{p^{2}}\right]
\end{aligned}
$$

and for $q=p=0$,

$$
\Omega^{\prime}(0,0,0)=\frac{\pi^{2}}{6} \quad \Omega^{\prime}(\pi, 0,0)=-\frac{\pi^{2}}{12} .
$$

\section{ACKNOWLEDGMENT}

The authors would very much like to thank the four reviewers of this paper. Their comments have added much to the quality and clarity of this paper and pointed the way to further research with respect to the combined use of the differential surface admittance concept and surface integral-equation techniques. The authors especially draw the attention of the readers to the first example given in Section VI-B and the subsequent discussion on the surface currents discretization, which the reviewers encouraged the authors to add to their original manuscript.

\section{REFERENCES}

[1] J. W. Bandler and M. Mongiardo, "Guest editorial," IEEE Trans. Microw. Theory Tech. (Special Issue), pt. 2, vol. 52, no. 1, pp. 241-244, Jan. 2004.

[2] F. Olyslager and D. De Zutter, "Skin effect," in Wiley Encyclopedia of Electrical and Electronics Engineering. New York: Wiley, 1999, vol. 19 , pp. 314-318.

[3] M. J. Tsuk and J. A. Kong, "A hybrid method for the calculation of the resistance and inductance of transmission lines with arbitrary cross sections," IEEE Trans. Microw. Theory Tech., vol. 39, no. 8, pp. 1338-1347, Aug. 1991.

[4] T. K. Sarkar and A. R. Djordjević, "Wideband electromagnetic analysis of finite conductivity cylinders," Progress Electromagn. Res., vol. 16, pp. 153-173, 1997.

[5] G. Antonini, A. Orlandi, and C. R. Paul, "Internal impedance of conductors of rectangular cross section," IEEE Trans. Microw. Theory Tech., vol. 47, no. 7, pp. 979-985, Jul. 1999.
[6] K. M. Coperich, A. Ruehli, and A. Cangellaris, "Enhanced skin effect for partial-element equivalent-circuit (PEEC) models," IEEE Trans. Microw. Theory Tech., vol. 48, no. 9, pp. 1435-1442, Sep. 2000.

[7] C. L. Holloway, "Edge and surface effects on conductor loss associated with planar circuits," Univ. Colorado at Boulder, Boulder, CO, MIMICAD Tech. Rep. 12, Apr. 1992.

[8] L. Knockaert, P. Van den Abeele, and D. De Zutter, "Surface impedance of cylinders and wedges: A Neumann approach," Int. J. Electron. Commun., vol. 53, no. 1, pp. 11-17, 1999.

[9] L. Friedlander, "Remarks on Dirichlet and Neumann eigenvalues," Amer. J. Math., vol. 117, no. 1, pp. 257-262, 1995.

[10] F. Olyslager, N. Faché, and D. De Zutter, "New fast and accurate line parameter calculation of general multiconductor transmission lines in multilayered media," IEEE Trans. Microw. Theory Tech., vol. 39, no. 6, pp. 901-909, Jun. 1991.

[11] R. E. Collin, Field Theory of Guided Waves, 2nd ed. Piscataway, NJ: IEEE Press, 1991.

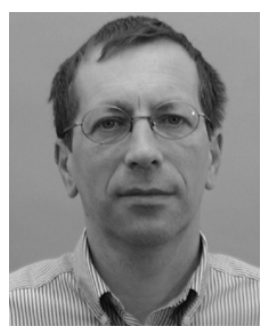

Daniël De Zutter (M'92-SM'96-F'01) was born in 1953. He received the M.Sc. degree in electrical engineering, $\mathrm{Ph} . \mathrm{D}$. degree, and a thesis leading to a degree equivalent to the French Aggrégation or the German Habilitation from Ghent University, Gent, Belgium, in 1976, 1981, and 1984, respectively.

From 1976 to 1984, he was a Research and Teaching Assistant with Ghent University. From 1984 to 1996, he was with the National Fund for Scientific Research of Belgium. He is currently a Full Professor of electromagnetics with the Department of Information Technology, Ghent University. Most of his earlier scientific work dealt with the electrodynamics of moving media. He has authored or coauthored over 135 international journal papers and 140 papers appearing in conference proceedings. He coauthored Electromagnetic and Circuit Modeling of Multiconductor Transmission Lines (Oxford, U.K.: Oxford Univ. Press, 1993). His current research focuses on all aspects of circuit and electromagnetic modeling of high-speed and high-frequency interconnections and packaging, electromagnetic compatibility (EMC), and numerical solutions of Maxwell's equations.

Dr. De Zutter was the recipient of the 1990 Montefiore Prize of the University of Liège, the 1995 IEEE Microwave Prize Award (with F. Olyslager and K. Blomme) presented by the IEEE Microwave Theory and Techniques Society (IEEE MTT-S) for the best publication in the field of microwaves, and the 1999 Transactions Prize Paper Award presented by the IEEE Electromagnetic Compatibility (EMC) Society.

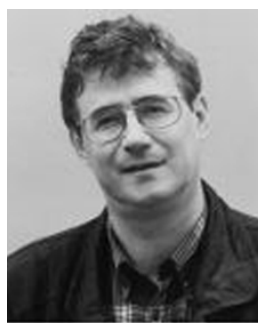

Luc Knockaert (M'81-SM'00) received the M.Sc. degree in physical engineering, M.Sc. degree in telecommunications engineering, and Ph.D. degree in electrical engineering from Ghent University, Gent, Belgium, in 1974, 1977, and 1987, respectively.

From 1979 to 1984 and from 1988 to 1995 , he was involved with North-South cooperation and development projects with the University of Congo (formerly Zaire) and the University of Burundi. He is currently a Guest Professor and Senior Researcher with the Department of Information Technology (INTEC)-Interuniversity Microelectronics Centre (IMEC), Leuven, Belgium. He has authored or coauthored approximately $50 \mathrm{SCI}$ peer-reviewed journal papers. His current interests are the application of statistical and linear algebra methods in signal identification, entropy applications, matrix compression, ROM, and computational electromagnetics.

Dr. Knockaert is a member of the Association for Computing Machinery (ACM) and the Society for Industrial and Applied Mathematics (SIAM). 